\title{
FIELDS OF FRACTIONS FOR GROUP ALGEBRAS OF FREE GROUPS
}

\author{
BY \\ JACQUES LEWIN(1)
}

\begin{abstract}
Let $K F$ be the group algebra over the commutative field $K$ of the free group $F$. It is proved that the field generated by $K F$ in any Mal'cev-Neumann embedding for $K F$ is the universal field of fractions $U(K F)$ of $K F$. Some consequences are noted. An example is constructed of an embedding $K F \subset D$ into a field $D$ with $D \neq U(K F)$. It is also proved that the generalized free product of two free groups can be embedded in a field.
\end{abstract}

I. Introduction. P. M. Cohn has recently shown [3, Chapter 7] that if $R$ is a semifir then there is an embedding of $R$ in a (not necessarily commutative) field $U(R)$ which is universal in the sense that if $R \subset D$ is another embedding of $R$ in a field then there is a specialization of $U(R)$ onto $D$ which extends the identity map of $R$. In particular, free associative algebras and free group algebras have universal fields of fractions.

Let now $F$ be a free group and $K$ a commutative field. I. Hughes [5] singles out a class of "free" embeddings (see definition below, \&II) of $K F$ into fields and shows that any two free embeddings which are both generated (qua fields) by $K F$ are $K F$-isomorphic This makes it plausible that $U(K F)$ is a free embedding and we show that this is indeed the case. Oddly enough this is not proved by verifying directly the freedom property of $U(K F)$, but by first proving a subgroup theorem: If $G$ is a subgroup of $F$, then $K G$ generates, in $U(K F)$, its universal field $U(K G)$.

The significance of our theorem is that it shows that $U(K F)$ is in fact the field generated by $K F$ in any Mal'cev-Neumann embedding of $K F[10]$. If $R$ is a free $K$-algebra on the generators of $F$, then it is easily seen that $U(R)=U(K F)$. Thus we have both $U(R)$ and $U(K F)$ represented in power series over $F$. This has several interesting consequences: $U(F)$ can be ordered; the center of $U(F)$ is $K$ (if $F$ is not commutative); there is a homomorphism of the multiplicative group $U(F)^{*}$ onto the free group $F$. ( $F$ is actually a retract of $U(F)^{*}$.)

Going back to groups, we show that any generalized free product $G$ of two free groups can be embedded in a field. However, using an example of M. Dunwoody [4], we show that there need not exist a fully inverting (definition below) embedding for $G$, even if the amalgamated subgroup is cyclic.

Hughes [5] asks whether there exists a nonfree embedding of the free group algebra $K F$. We close by exhibiting such an embedding.

Received by the editors December 11, 1972.

AMS (MOS) subject classifications (1970). Primary 16A26, 20E05, 16A06.

Key words and phrases. Free algebras, free group algebras, universal fields of fractions, generalized free products of free groups.

(1) This work was supported by NSF grant GP 33050. 
II. $U(K F)$ is Hughes-free. If $R$ is a semifir, we denote by $U(R)$ the universal field of fractions of $R . R \subseteq U(R)$ is fully inverting: every full $R$-matrix inverts over $U(R)$. Every element $u_{1}$ of $U(R)$ is rational over $R$, i.e. $u_{1}$ is the first component of a solution $\mathbf{u}$ of a matrix equation $A \mathbf{u}+\mathbf{a}=0$ where $A$ is a full $n \times n R$-matrix and a $\in R^{n}$. (Recall that $A$ is full if $A$ is not a product of two matrices of smaller size.) If $S$ is a subring of $R$, the inclusion $S \rightarrow R$ is honest if every full $S$-matrix is still full as an $R$-matrix.

If $h: R \rightarrow D$ is a homomorphism into a field $D$, then there is universal specialization $p: U(R) \rightarrow D$ which extends $h$. The domain of $p$ consists of the set of entries of inverses of those $R$-matrices whose image is invertible over $D$.

Details and proofs may be found in Cohn [3, Chapter 7].

In particular, if $F$ is a free group and $K$ a commutative field, then $K F$ is a semifir so the above results apply. We write $U(F)$ for $U(K F)$.

If $H$ is a sugroup of $F$ and $K F$ is embedded in a field $D$, we denote by $\operatorname{Div}_{D}(H)$ the smallest subfield of $D$ which contains $H$ and $K$. Note that $\operatorname{Div}_{D}(H)$ is rational over $\boldsymbol{H}$.

Our aim is to show that for any subgroup $G$ of a free group $F, \operatorname{Div}_{U(F)}(G)$ $=U(G)$.

The universal specialization from $U(G)$ into $U(F)$ will be a monomorphism $U(G) \rightarrow U(F)$ provided the inclusion $K F \rightarrow K G$ is honest. It is this that we shall prove. We first deal with a special case.

Lemma 1. Let $F$ be a free group and $G$ a normal subgroup of finite index in $F$. Then the inclusion $K G \rightarrow K F$ is honest.

Proof. Let $s_{1}=1, s_{2}, \ldots, s_{n}$ be a set of coset representatives for $G$ in $F$. Then $K F=\bigoplus_{i=1}^{n}(K G) s_{i}$. Right multiplication by an element of $K F$ is a left $K F$, and hence a left $K G$, module homomorphism. Thus we have a faithful representation $\varphi: K F \rightarrow(K G)_{n}$, the $n \times n$ matrices over $K G$.

If $v \in K G$, then $s_{i} v=s_{i} v s_{i}^{-1} \cdot s_{i}=v^{s_{i}} \cdot s_{i}$. Since $G$ is normal in $F, v^{s_{i}} \in K G$. Thus $v \varphi$ is the diagonal matrix

$$
v \varphi=\left[\begin{array}{llllll}
v & & & & & 0 \\
& v^{s_{1}} & & & \\
& & \cdot & & \\
& & & \cdot & \\
& & & \cdot & \\
0 & & & & v^{s_{n}}
\end{array}\right] .
$$

In the obvious way, we extend $\varphi$ to matrices over $K F$. Let now $M$ be a $K G$-matrix which is full over $K G$. Since conjugation by $s_{i}$ is an automorphism of $K G, M^{s_{i}}$ is also full over $K G$ for $i=1, \ldots, n$. $K G$ is again a free group algebra, so $K G$ is a fir. It follows [3, Theorem 6.4, p. 282] that the diagonal sum $N=M+M^{s_{1}}+\cdots$ 
$\dot{+} M^{s_{n}}$ is full. But, by (1), $M \varphi$ is similar, via a permutation matrix, to $N$. So $M$, as a $K F$-matrix, maps under $\varphi$ to a full matrix. So $M$ is full over $K F$ and the lemma is proved.

The next step is to drop the normality assumption on $G$. We first need an easy lemma.

If $S$ is a subset of a $K$-algebra, denote by $K\langle S\rangle$ the subalgebra it generates.

Lemma 2. Let $G$ be a normal subgroup of finite index in the group $F$, and suppose $K F$ is embedded in a field $D$. Then $\operatorname{Div}_{D}(F)$ has finite dimension as a left $\operatorname{Div}_{D}(G)$ vector space. Further $\operatorname{Div}_{D}(F)=K\left\langle\operatorname{Div}_{D}(G), F\right\rangle$.

Proof. Let $s_{1}=1, s_{2}, \ldots, s_{n}$ be a set of coset representatives for $G$ in $F$, and consider $A=\bigoplus_{i=1}^{n} \operatorname{Div}_{D}(G) s_{i}$. Now $s_{i}$ induces, by conjugation, an automorphism of $\operatorname{Div}_{D}(F)$ which leaves $G$, and hence $\operatorname{Div}_{D} G$, invariant. Thus for $d \in \operatorname{Div}_{D}(G)$, $d^{s_{i}} \in \operatorname{Div}_{D}(G)$. Thus since $s_{i} d=d^{s_{i}} s_{i}$ and $s_{i} s_{j}=g_{i j} s_{k}$ for some $k$ and some $g_{i j} \in G, A$ is a $K$-algebra. Since $A$ has finite left $\operatorname{Div}_{D}(G)$ dimension, and $A$ is an integral domain, $A$ is a field. Since $A$ contains $F, A=\operatorname{Div}_{D}(F)$. Clearly $A$ is generated by $\operatorname{Div}_{D}(G)$ and $F$.

Lemma 3. Let $F$ be a free group, and $L$ a subgroup of finite index in $F$. Then $\operatorname{Div}_{U(F)}(L)=U(L)$.

Proof. Let $G$ be the intersection of the conjugates of $L$. Then $G$ is still of finite index in $F$ and is of course normal. Then, by Lemma $1, \operatorname{Div}_{U(F)}(G)=U(G)$ and $\operatorname{Div}_{U(L)}(G)=U(G)$.

Let $p$ be the universal specialization $p: U(L) \rightarrow \operatorname{Div}_{U(F)}(L)$. Since a full $K G$ matrix inverts over $U(G)$ it inverts over both $U(L)$ and $\operatorname{Div}_{U(F)}(L)$. So the entries of inverses of full $K G$-matrices are in the domain $\mathcal{L}$ of $p$. So $U(G) \subseteq \complement$. Since $p$ is an $L$-specialization, also $L \subseteq$. So, by Lemma $2, U(L)=K\langle U(G), L\rangle \subseteq \varrho$. Since $p$ is onto $\operatorname{Div}_{U(F)} L, p$ is an $L$-isomorphism $\operatorname{Div}_{U(F)} L \simeq U(L)$.

The next two lemmas allow us to go from subgroups of finite index to arbitrary finitely generated subgroups.

Lemma 4. Suppose the free group $F$ is the free product $H_{1} * H_{2}$ of two subgroups. Then $\operatorname{Div}_{U(F)}\left(H_{1}\right)=U\left(H_{1}\right)$.

Proof (P. M. Cohn). Embed $K H_{1}{ }^{*} K H_{2}$ in $U\left(H_{1}\right){ }^{*} U\left(H_{2}\right)$. If $M$ is a $K H_{1^{-}}$ matrix which is full over $K H_{1}$, then $M$ inverts over $U\left(H_{1}\right)$ and hence over $U\left(H_{1}\right) * U\left(H_{2}\right)$. So $M$ is full over $K H_{1} * K H_{2}=K F$. So $M$ inverts over $U(F)$ and hence inverts over $\operatorname{Div}_{U(F)}\left(H_{1}\right)$.

Lemma 5. Let $H$ be a finitely generated subgroup of the free group $F$. Then $H$ is a free factor in a subgroup $L$ of finite index in $F$.

A proof may be found in [6]. 
Theorem 1. If $H$ is a subgroup of the free group $F$, then $\operatorname{Div}_{U(F)}(H)=U(H)$.

Proof. If $H$ is finitely generated, this is the contents of Lemmas 3, 4 and 5. Let now $H$ be an arbitrary subgroup of $F$, and let $M$ be a $K H$-matrix which is full over $K H$. Then there is a finitely generated subgroup $H^{\prime}$ of $H$ such that $M$ is a matrix over $K H^{\prime} . M$ is still full over $K H^{\prime}$. Thus, by the finitely generated case, $M$ inverts over $U(F)$, and hence over $\operatorname{Div}_{U(F)}(H)$.

Recall that a group $G$ is indexed at $(H, t)$ if $H$ is normal in $G$ and $G / H$ is the infinite cyclic group generated by the coset $t H$. Let $F$ be a free group and $K F \subset D$ an embedding of $K F$ in a field. I. Hughes [5] makes the following definition: the embedding $K F \subset D$ is free if for any finitely generated subgroup $G$ of $F$, and indexing $(H, t)$ of $G$, then the powers of $t$ are left $\operatorname{Div}_{D}(K H)$ independent. He then shows

Hughes' theorem. If $K F \subset D_{1}, K F \subset D_{2}$ are two free embeddings of $K F$ then there is a $K F$-isomorphism $\operatorname{Div}_{D_{1}}(K F) \approx \operatorname{Div}_{D_{2}}(K F)$.

Proposition 6. $K F \subset U(F)$ is a Hughes-free embedding.

Proof. Let $G$ be a finitely generated subgroup of $F,(H, t)$ an indexing of $G$. Since $H$ is normal in $G$, conjugation by $t$ induces an automorphism $\tau$ of $U(H) \subseteq U(F)$. Form the skew Laurent polynomial ring $P=U(H)\left[z, z^{-1}\right]$ with the commutation rule $d z=z(d \tau)$. Then $P$ is an Ore domain with quotient field $D$. Since $P$ is a principal ideal domain, the embedding $P \subset D$ is fully inverting so that $D=U(P)$.

Now we have a homomorphism $\varphi: P \rightarrow K\left\langle U(H), t, t^{-1}\right\rangle$ which maps $z$ to $t$ and hence, composing with the inclusion $K\left\langle U(H), t, t^{-1}\right\rangle \rightarrow U(G)$, a homomorphism $h: P \rightarrow U(G)$.

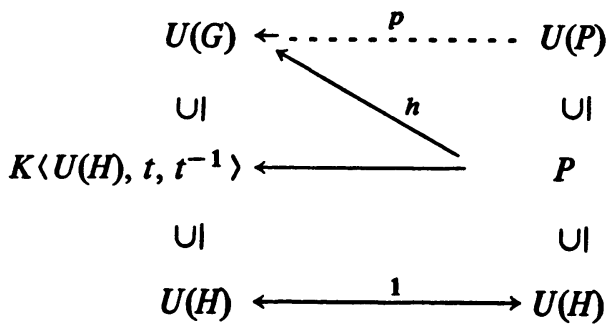

$h$ then extends to a specialization $p: U(P) \rightarrow U(G)$. But, identifying $K G$ $=K\left\langle H, t, t^{-1}\right\rangle$ with $K\left\langle H, z, z^{-1}\right\rangle, p$ is a $K G$-specialization. Since $U(G)$ is fully inverting for $K G$, so is $U(P)$ (Cohn [3, Theorem 2.3, p. 257]). Hence $U(G)$ and $U(P)$ are $K G$ isomorphic. Clearly, a $K G$-isomorphism is the identity on $H$, and hence maps $U(H)$ onto itself. Since $\left\{z^{i}\right\}$ is left $U(H)$ independent, $\left\{t^{i}\right\}$ is left $U(H)$ independent and the proposition is proved.

III. A series representation and applications. We first note that if $R=K\langle X\rangle$ is the free $K$-algebra on a set $X$ and $F$ is the free group on $X$, then $U(R)=U(F)$; 
since $x \in X$ has an inverse in $U(R)$, there is a homomorphism $K F \rightarrow U(R)$ which is the identity on $R$. This homomorphism extends to a specialization $p: U(F) \rightarrow U(R)$. So every matrix over $R$ which inverts over $U(R)$ inverts over $U(F)$, i.e. every full $R$-matrix inverts over $U(F)$. Since $U(F)$ is generated by $R, p$ is an isomorphism.

Recall the Mal'cev-Neumann method for embedding a free group algebra $K F$ in a field [10]: order $F$ and let $D$ be the set of formal series over $F$, with coefficients in $K$, whose support is well ordered. If $0 \neq p \in D$, then $p$ can be written uniquely as $p=k f\left(1+p^{\prime}\right), k \in K, f \in F, p^{\prime}=0$ or $p^{\prime}$ with positive support. Then $p^{-1}=\left(1-p^{\prime}+p^{\prime 2}-\cdots\right) g^{-1} k^{-1}$. Thus if $H$ is a subgroup of $F$, $\operatorname{Div}_{D}(H)$ consists of power series whose support is in $H$. This makes it clear that $D$ is Hughes-free. Thus, applying Hughes' theorem and the proposition, we obtain

Theorem 2. Let $F$ be a free group on the set $X, R=K\langle X\rangle$ the free $K$-algebra on $X ; D$ any Mal'cev-Neumann embedding of $F$. Then $U(R)=U(F) \simeq \operatorname{Div}_{D}(F)$.

Theorem 3. If $R$ is a free algebra over the ordered (commutative) field $K$, then $U(R)$ can be ordered.

Proof. We need only note that a Mal'cev-Neumann field can be ordered if $K$ can be.

Theorem 4 (cf. Klein [8]). If $R$ is a noncommutative free $K$-algebra, then the center of $U(R)$ is $K$.

Proof. Let $R$ be freely generated by $X,|X|>1$, and let $F$ be the free group on $X$. We may consider $U(R)$ as embedded in a Mal'cev-Neumann field for $F$. Let $z=\sum_{f \in F} k_{f} f$ be in the center of $U(R)$, and say $f_{1}$ is the least element in the support of $z$. Then, for $x \in X, x f_{1}$ and $f_{1} x$ are the least elements in the supports of $x z$ and $z x$. So $f_{1}$ is in the center of $F$, i.e. $f_{1}=1$. So $k_{1}-z$ is again in the center of $U(R)$. But then $k_{1}-z=0$ or its support consists of positive elements. This last leads to a contradiction and hence $z \in K$.

Theorem 5. Iot $R$ be a free $K$-algebra, $F$ the corresponding free group, and let $U(R)^{*}$ be the multiplicative group of nonzero elements of $U(R)$. Then the free group $F$ is a retract of $U(R)^{*}$.

Proof. We regard $U(R)$ as embedded in a Mal'cev-Neumann field for $F$. Let $N$ be the subset of $U(R)^{*}$ of elements $k+P$, where $0 \neq k \in K$, and $P=0$ or $P$ has positive support. An easy calculation shows that $N$ is normal in $U(R)^{*}$. If $g_{1}, g_{2}$ are different elements of $F$, then $g_{1} g_{2}^{-1} \neq 1$. So $g_{1} g_{2}^{-1} \notin N$ and thus $g_{1} \neq g_{2} \bmod N$. Also, if $Q \in U(R)^{*}, Q$ can be written uniquely as $Q=$ $g k\left(1+Q^{\prime}\right)$ with $k\left(1+Q^{\prime}\right) \in N, g \in F$. Then $Q=g \bmod N$ and $Q \rightarrow g$ is the required retraction of $U(R)^{*}$ onto $F$.

Corollary. Let $U(R)_{a b}^{*}$ be the commutator factor group of $U(R)^{*}$. The projection $U(R)^{*} \rightarrow U(R)_{a b}^{*}$ is injective on the generators of $R$. 
(This provides a partial answer to problem 10 on p. 286 of [3].)

IV. Generalized free products of free groups. Recall from [3] the following fundamental property of free products of rings over a (skew) field $D$. Let $R_{1}, R_{2}$ be $D$-rings and let $\{1\} \cup S_{i}$ be a left $D$-basis for $R_{i}$. Then the monomials on $S_{1} \cup S_{2}$, no two successive letters of which are in the same factor, form, together with 1 , a left $D$-basis for the free product $R_{1}{ }^{*} R_{D}$.

Theorem 6 (cf. [1, Corollary 3.1], [7, Theorem 9], [9]). Let $F_{1}, F_{2}$ be two free groups with a common subgroup $H$ and let $G$ be the generalized free product $F_{1}{ }_{H} F_{2}$. Then the group algebra $K G$ can be embedded in a field.

Proof. If $\{1\} \cup S_{i}$ is a left transversal for $H$ in $F_{i}$ then it is clear by looking in Mal'cev-Neumann fields that $\{1\} \cup S_{i}$ is a left $\operatorname{Div}_{U\left(F_{i}\right)}(H)$-independent set. Further there are $K H$ isomorphisms $\operatorname{Div}_{U\left(F_{1}\right)}(H) \simeq \operatorname{Div}_{U\left(F_{2}\right)}(H) \simeq U(H)$. These observations and the remark preceding the theorem show that the free product $R=U\left(F_{1}\right){ }^{*}{ }_{U(H)} U\left(F_{2}\right)$ makes sense and embeds $K G$. But $R$ is a free ideal ring [2] and so has a universal field of fractions $U(R)$. Thus $K G \subseteq U(R)$.

Unfortunately, $U(R)$ need not be fully inverting for $K G$. Indeed $K G$ need not have a fully inverting embedding. For Dunwoody [4] has shown that if $G=\left\langle a, b ; a^{2}=b^{3}\right\rangle$, then $K G$ has a nonfree finitely generated projective module $P$. Such a ring has a full matrix which is not invertible in any field which embeds it: let $M$ be a free module of least rank such that $M=P_{1} \oplus P$. The projection $M \rightarrow P$ is given by an idempotent matrix $\mu$ which is not the identity. Thus $\mu$ does not become invertible in any overfield. However, $\mu$ is full. For otherwise $P \subseteq N$, a submodule of $M$ with fewer generators. Since $P$ is a direct summand of $M$, it is a direct summand of $N$, and hence needs fewer generators than $M$, contradicting the minimality of the rank of $M$.

V. An example. We now construct a nonfree embedding of a free group algebra in a field.

Let $F$ be the free group $F_{1} * F_{2}$ where $F_{1}$ is free on $z$ and $F_{2}$ is free on $x$ and $w$. We embed $k F_{i}$ in $R_{i}=U\left(F_{i}\right)$. In $R_{1}$ we choose a $K$-basis $\{1\} \cup S_{1}$ such that $\left\{(1+z)^{-1}, z^{i} ; i= \pm 1, \pm 2, \ldots\right\} \subset S_{1}$ and in $R_{2}$ we choose a $K$-basis $\{1\} \cup S_{2}$ with $F_{2} \backslash\{1\} \subset S_{2}$. Let $b=(1+z)^{-1}$. In $R=R_{1}{ }^{*} R_{2}$ let $T^{\prime}$ be the set of elements $r=f_{1} b f_{2} b \cdots f_{n} b f_{n+1}$ where $f_{i} \in F$ is a reduced word which neither begins or ends with $z^{ \pm 1}$ for $i=2, \ldots, n-1, f_{1}=1$ or $f_{1}$ does not end in $z^{ \pm 1}, f_{2}=1$ or $f_{2}$ does not begin with $z^{ \pm 1}$. We extend the length function $l$ of $F$ to a length function on $T=T^{\prime} \cup F \backslash\{1\}$ by declaring $l(r)=n+\sum_{i=1}^{n+1} l\left(f_{i}\right)$. It is clear that a set of elements of $T$ that are spelled differently is left $K$-independent. We embed $R$ in its universal field $U(R)$. In $U(R)$ we may choose a basis $\{1\} \cup S$ with $T \subset S$. Let $u=b w(1+x)$, and let $Q=\operatorname{Div}_{U(R)}(k[u])$. We claim that in $U(R)$ the set $F$ is left $Q$-independent. 
We first note that $Q$ is the field of right quotients of $k[u]$ so that a set is left $Q$ independent if and only if it is $k[u]$-independent. So we need only show that $F$ is left $k[u]$-independent. We note next that $b w$ and $b w x$ freely generate a free subalgebra of $U(R)$. Let then $f_{\alpha}$ be distinct elements of $F$ and suppose that there are polynomials $p_{\alpha}(u)$, not all zero with $\sum p_{\alpha}(u) f_{\alpha}=0$. Choose $\alpha$ such that $p_{\alpha}(u)$ has maximal $u$ degree, say $n$, and $f_{\alpha}$ has maximal length among the $f_{\beta}$ for which $p_{\beta}(u)$ has degree $n$. Two cases arise.

1. $f_{\alpha}$ does not begin with $x^{-1}$. Then $p_{\alpha}(u) f_{\alpha}$ gives rise to a term $t$ $=(b w x)^{n-1} b w x f_{\alpha}$. This term has length $4 n+l\left(f_{\alpha}\right)$ and has degree $n$ on $b$. Also it is clear that this length is maximal among the monomials in the expansion of $\sum p_{\alpha}(u) f_{\alpha}$ which have degree $n$ on $b$. So since the sum vanishes, for some $\beta \neq \alpha, p_{\beta}(u)$ has degree $n$ and the term $t$ also appears in the expansion of $u^{n} f_{\beta}$. Since $f_{\alpha}$ had maximal length, this forces $f_{\alpha}=f_{\beta}$, a contradiction.

2. We may then assume that $f_{\alpha}$ starts with $x^{-1}$. Now $p_{\alpha}(u) f_{\alpha}$ gives rise to a term $(b w x)^{n-1} b w f_{\alpha}$ of length $4 n+l\left(f_{\alpha}\right)-1$, and this is the only term of this length in the expansion of $p_{\alpha}(u) f_{\alpha}$ (since all other terms in the expansion of $p_{\alpha}(u) f_{\alpha}$ either end with $x$ or are too short). It is again easy to see that this implies that $f_{\beta}=f_{\alpha}$ for some $\alpha \neq \beta$, and this contradiction proves the claim.

We now provide ourselves with another copy $U(R)^{\prime}$ of $U(R)$ and consider the free product $V=U(R) * U(R)^{\prime}$ amalgamating $Q$ with $Q^{\prime}$. Then the group words on the letters $z, x, w, z^{\prime}, x^{\prime}, w^{\prime}$ are left $Q$-independent. and hence $K$-independent. Thus the group $G$ generated by these letters is free on them and the $K$-algebra generated by $G$ in $U(V)$ is the group algebra $K G$. Now $V$ is still a free ideal ring [2] and hence we may embed $V$ in $U(V)$. Clearly $G$ generates $U(V)$ qua skew fields. However, in $U(V)$,

$$
(1+z)^{-1} w(1+x)=\left(1+z^{\prime}\right)^{-1} w^{\prime}\left(1+x^{\prime}\right)
$$

so that

$$
x=w^{-1}(1+z)\left(1+z^{\prime}\right)^{-1} w^{\prime}\left(1+x^{\prime}\right)-1 .
$$

Hence $\operatorname{Div}\left(g p\left(x, z, w, x^{\prime}, z^{\prime}, w^{\prime}\right)\right)=\operatorname{Div}\left(g p\left(z, w, x^{\prime}, z^{\prime}, w^{\prime}\right)\right)$ and $U(V)$ does not distinguish $G$ from a free factor of $G$. However, it is clear by looking in a Mal'cev-Neumann embedding of $K G$ that if $H_{1}$ and $H_{2}$ are distinct subgroups of $G$, then $\operatorname{Div}_{U(G)}\left(H_{1}\right) \neq \operatorname{Div}_{U(G)}\left(H_{2}\right)$. Thus $G \subseteq U(V)$ is not a free embedding.

\section{REFERENCES}

1. G. Baumslag, Positive one relator groups, Trans. Amer. Math. Soc. 156 (1971), 165-183. MR 43 \#325.

2. P. M. Cohn, Free ideal rings, J. Algebra 1 (1964), 47-69; correction ibid. 6 (1967), 410. MR 28 \# 5095; 35 \#214.

3.- Free rings and their relations, Academic Press, New York and London, 1971.

4. M. Dunwoody, Relation modules,

Soc. 4 (1972), 151-155. 
5. I. Hughes, Division rings of fractions for group rings, Comm. Pure Appl. Math. 23 (1970), 181-188. MR 41 \#8533.

6. A. Karrass and D. Solitar, On finitely generated subgroups of a free group, Proc. Amer. Math. Soc. 22 (1969), 209-213. MR 39 \#6961.

7.—, The subgroups of a free product of two groups with an amalgamated subgroup, Trans. Amer. Math. Soc. 150 (1970), 227-255. MR 41 \#5499.

8. A. Klein, Involutorial division rings with arbitrary center, Proc. Amer. Math. Soc. 34 (1972), $38-42$.

9. J. Lewin, A note on zero divisors in group rings, Proc. Amer. Math. Soc. 31 (1972), 357-359.

10. B. H. Neumann, On ordered division rings, Trans. Amer. Math. Soc. 66 (1949), 202-252. MR 11, 311.

Department of Mathematics, Syracuse University, Syracuse, New York 13210 